\title{
Coherent optical control of acoustic phonon oscillations in InGaN/GaN multiple quantum wells
}

\author{
Chi-Kuang Sun, ${ }^{\text {a) }}$ Yue-Kai Huang, and Jian-Chin Liang \\ Department of Electrical Engineering and Graduate Institute of Electro-Optical Engineering, \\ National Taiwan University, Taipei, Taiwan, 10617 Republic of China

\begin{abstract}
Amber Abare and Steven P. DenBaars
Department of Electrical and Computer Engineering, University of California, Santa Barbara, California 93106
\end{abstract}

(Received 1 June 2000; accepted for publication 4 January 2001)

\begin{abstract}
We demonstrate coherent optical control of phonon oscillations using a femtosecond control pulse. The experiments were performed in InGaN/GaN multiple quantum wells. Coherent acoustic phonon oscillations was initiated with an UV femtosecond pulse. The subsequent manipulation, including magnitude and phase, of the coherent acoustic phonon oscillations was achieved using another UV femtosecond pulse by controlling the pulse time delay and intensity. (C) 2001 American Institute of Physics. [DOI: 10.1063/1.1350598]
\end{abstract}

Progress in femtosecond lasers and ultrafast spectroscopy technology has enabled us to generate and directly observe coherent oscillations of phonon modes. ${ }^{1-14}$ Oscillation changes in optical properties have been observed in GaAs, ${ }^{1-3}$ $\mathrm{Ge}^{4}{ }^{4}$ and a number of other materials. ${ }^{2,5}$ These optically excited coherent optical phonon modes behave like classical oscillators and have nonzero time-dependent displacements. ${ }^{6}$ This is different from spontaneous phonon emission, which is an incoherent process that populates the phonon modes with the expectation value of each mode being zero. For acoustic phonons, semiconductor superlattices exhibit zone folding of the acoustic branches within the mini-Brillouin zone enabling the observation of coherent oscillations of the zone-folded acoustic phonons in AlAs/GaAs superlattices. ${ }^{7-9}$ However, the observed reflection modulation was extremely small. Acoustic vibrations of nanoparticles ${ }^{10-12}$ were also recently observed through impulsive excitation of their fundamental expansion modes. The oscillation damping was dominated by fast energy loss from the nanoparticles to their surrounding media. Recently, we have reported the generation of coherent acoustic phonon oscillations in $\mathrm{InGaN} / \mathrm{GaN}$ multiple quantum wells (MQWs) with strain-induced piezoelectric field. ${ }^{13}$ Strong oscillations can be easily generated with induced absorption modulation on the order of $10^{-2}-10^{-3}$. The oscillation frequency, on the order of terahertz, can be linearly varied by changing the sample period width. The uncertainty of carrier distribution wave vectors due to finite sample width was found to determine the observed oscillation dephasing time. ${ }^{14}$ With 14 quantum wells, dephasing time on the order of $10 \mathrm{ps}$ was observed.

In this letter, we demonstrate optical control of the coherent acoustic phonon oscillations using a separate optical (control) pulse. The concept of coherent control was previously applied to many interesting fields including molecular motion manipulation, ${ }^{15}$ electronic quantum dynamics control, ${ }^{16,17}$ electron-phonon scattering control, ${ }^{18}$ photocurrent generation in semicondcutors, ${ }^{19}$ and charge oscillation

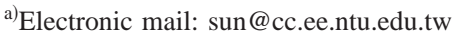

control with terahertz radiation. ${ }^{20}$ We extended the concept of coherent optical control to the manipulation of coherent phonon oscillations. Strong magnitude and relatively long dephasing time of the coherent acoustic phonon oscillation in the InGaN/GaN MQW system make it suitable for this study.

Optical control experiments were performed at room temperature on 14 period InGaN/GaN MQWs. ${ }^{13}$ Because of the large piezoelectric constants along the [0001] orientation in group-III nitrides, a strain-induced piezoelectric field on the order of megavolts per centimeter is expected. The InGaN/GaN MQW samples were grown on $c$-plane sapphire using atmospheric pressure metalorganic chemical vapor deposition. After annealing the substrate at $1050{ }^{\circ} \mathrm{C}$, a 19 nm-thick GaN substrate was deposited at $525^{\circ} \mathrm{C}$. The temperature was then raised to $1080^{\circ} \mathrm{C}$ to grow $\mathrm{GaN}$ of $2.5 \mu \mathrm{m}$ thickness. The 14 period $\mathrm{In}_{0.1} \mathrm{Ga}_{0.9} \mathrm{~N} / \mathrm{GaN} \mathrm{MQW}$ was then grown on this thick GaN layer at a temperature of $790^{\circ} \mathrm{C}$. The barrier width was $43 \AA$ while a well width of either 50 or $62 \AA$ was deposited. The sample was finished with a 0.1$\mu$ m-thick $\mathrm{Al}_{0.1} \mathrm{Ga}_{0.9} \mathrm{~N}$ cap layer. Room temperature absorption measurements indicated that the band gaps of the MQWs were around $422 \mathrm{~nm}(2.92 \mathrm{eV})$ and $430 \mathrm{~nm}(2.88 \mathrm{eV})$ for 50 and $62 \AA \mathrm{MQWs}$, respectively. The crystal structure was wurzite.

The experiments were performed using a Kerr-lensmode-locked Ti:sapphire laser with an $82 \mathrm{MHz}$ repetition rate. The experimental setup is shown schematically in Fig. 1. The laser output pulses were frequency doubled using a BBO crystal to reach band gap energy of the InGaN/GaN MQWs. The frequency-doubled pulses had a pulse width of $180 \mathrm{fs}$ at a wavelength of $390 \mathrm{~nm}$ measured by a two-photonabsorption-type pump-probe autocorrelation in a bulk GaN thin film. ${ }^{21}$ The femtosecond pump pulses photoexcited carriers in the MQWs. Because of the periodic distribution of the photoexcited carriers, carrier population with a wavevector $q=2 \pi / a$, where $a$ is the MQW period width (93 or 105 $\AA$ ), was thus created. Coherent oscillation, of the selected acoustic phonon mode (with wave vector $q$ ), was then initiated. ${ }^{14}$ With a new equilibrium position due to the pho- 


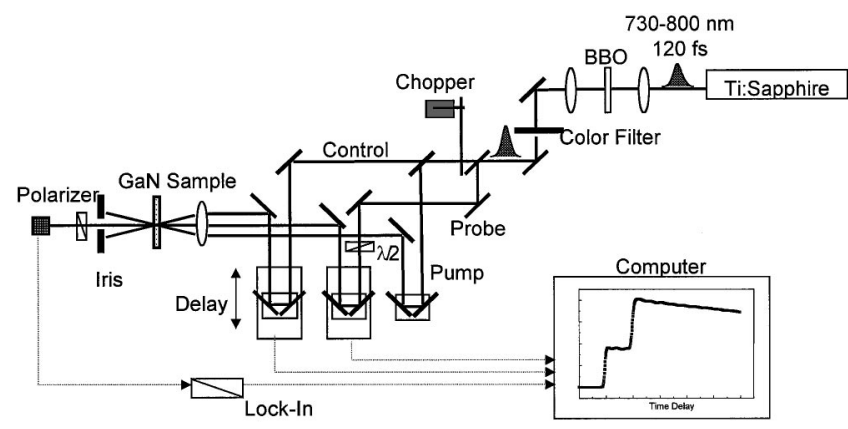

FIG. 1. Schematics of the experimental setup.

tocarrier screening effect, displacive cosine oscillations of the coherent acoustic phonons was induced. With a space charge field much smaller than the built-in piezoelectric field, the displacive force was proportional to the photogenerated excess carrier density. ${ }^{14}$ After initiation of the oscillation, an UV control pulse was directed into the same area to perform the oscillation manipulation with time delay controlled by a $0.1 \mu \mathrm{m}$ stepping stage. The relative pump/ control pulse intensities were managed through adjustable neutral-density filters. In order to have a maximum spatial overlap between the pump and control pulses, the incident angles of these two pulses were carefully tuned so that they were identical. The generated and manipulated acoustic phonon oscillations modulated the MQW absorption spectrum through quantum-confined Franz-Keldysh effect, ${ }^{14}$ and was detected with a weak probe pulse by measuring the probe transmission change as a function of probe pulse time delay.

Figure 2 shows an example of the measured transient transmission change due to the pump and control pulses for the $50 \AA \mathrm{MQW}$ sample at a wavelength of $390 \mathrm{~nm}$. Incident pump power was $4.6 \mathrm{~mW}$ with a corresponding optical fluence of $3.6 \times 10^{-5} \mathrm{~J} / \mathrm{cm}^{2}$. By measuring the reflected and transmitted optical power before and after the sample, pumpinduced two-dimensional (2D)/three-dimensional (3D) carrier densities were estimated to be $\sim 2.4 \times 10^{12} \mathrm{~cm}^{-2} / 4.7$ $\times 10^{18} \mathrm{~cm}^{-3}$ with a measured focal spot diameter of $14 \mu \mathrm{m}$. After pump excited carriers at zero time delay, a cosinusoidal acoustic phonon oscillations with a period of 1.38 ps can be observed in the transient transmission measurements, on top of a large positive background due to the bandfilling effect. ${ }^{13}$ The magnitude and phase of the coherent oscillation were

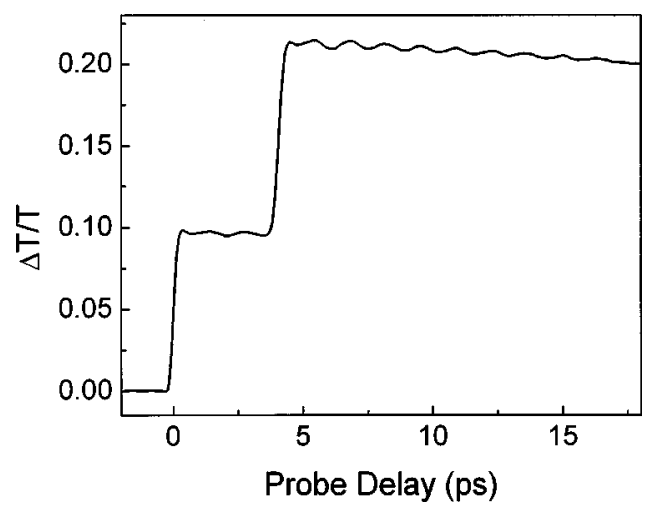

FIG. 2. Transmission change vs probe delay with both pump and control pulses in the $50 \AA$ InGaN MQW sample. The excitation wavelength was $390 \mathrm{~nm}$.

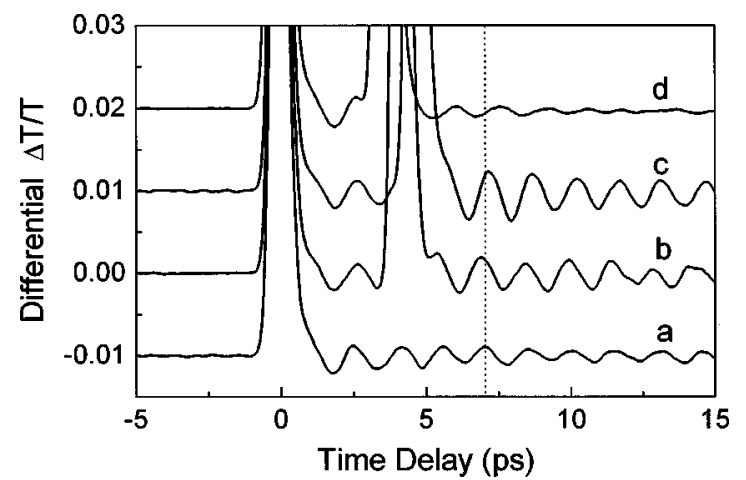

FIG. 3. The differentiated transmission changes versus probe delay with (b), (c), (d) and without (a) the control pulse. The experiments were performed in the $62 \AA$ InGaN MQW sample. Oscillation phase shifts of -20 [trace (b)], +35 [trace (c)], and +135 [trace (d)] degrees were achieved with the control pulses.

then manipulated using a followed control pulse, with controlled optical intensity and time delay. For the example shown in Fig. 2, the control pulse arrived at 4 ps after zero time delay and induced another transmission background increase, indicating absorption of control pulse energy. The incident control power inside the sample was $5.7 \mathrm{~mW}$ (optical fluence $\sim 4.5 \times 10^{-5} \mathrm{~J} / \mathrm{cm}^{2}$ ) with an induced $2 \mathrm{D} / 3 \mathrm{D}$ photocarrier density $\sim 2.6 \times 10^{12} \mathrm{~cm}^{-2} / 5.2 \times 10^{18} \mathrm{~cm}^{-3}$ under the existence of pump-induced carriers. Due to imperfect pulse overlap and absorption modification with pumpinduced carriers, it is less accurate to estimate the displacive force ratio using control/pump optical fluence. We estimated the displacive force ratio exerted by the control and pump pulses using their induced positive step responses as shown in Fig. 2. Due to the bandfilling effect, the magnitudes of the positive transmission increases induced by the control/pump pulses were proportional to the deposited carrier densities in the probed MQW region, which reflected the relative displacive forces. The bandfilling step ratio of control and pump pulses in Fig. 2 is 1.15, in close agreement with our estimated photocarrier density ratio $(\sim 1.1)$, indicating excellent pump/control pulse overlap in the probing region.

For easier study of the oscillation magnitude and phase variation, we differentiated the obtained transmission traces. Figure 3 shows typical differentiated transmission signals without [trace (a)] and with the control pulse [traces (b), (c), (d)] performed in the $62 \AA \mathrm{MQW}$ sample at a wavelength of $395 \mathrm{~nm}$. The average incident pump power inside the sample was $4.5 \mathrm{~mW}$ (optical fluence $\sim 3.6 \times 10^{-5} \mathrm{~J} / \mathrm{cm}^{2}$ ) with estimated 2D/3D photocarrier densities of $3 \times 10^{12} \mathrm{~cm}^{-2} / 5$ $\times 10^{18} \mathrm{~cm}^{-3}$. The optical fluence of the control pulse was 1.31 times of the pump pulse (optical fluence $\sim 4.7$ $\times 10^{-5} \mathrm{~J} / \mathrm{cm}^{2}$ ), with an estimated (measured) carrier density 1.28 times that of the pump pulse due to the absorption modulation effect. The bandfilling step ratio between control and pump was around 1.2. Due to a different sample period width, an acoustic phonon oscillation period of 1.54 ps was observed in the $62 \AA$ sample. ${ }^{13}$ For traces (b), (c), (d) of Fig. 3 , phase shifts of $-20,+35$, and +135 degrees were demonstrated by exciting with the control pulses at delay time corresponding to $-90(4.24 \mathrm{ps}),+90(5.0 \mathrm{ps})$, and +160 (3.76 ps) degrees of the first induced cosine oscillations. 

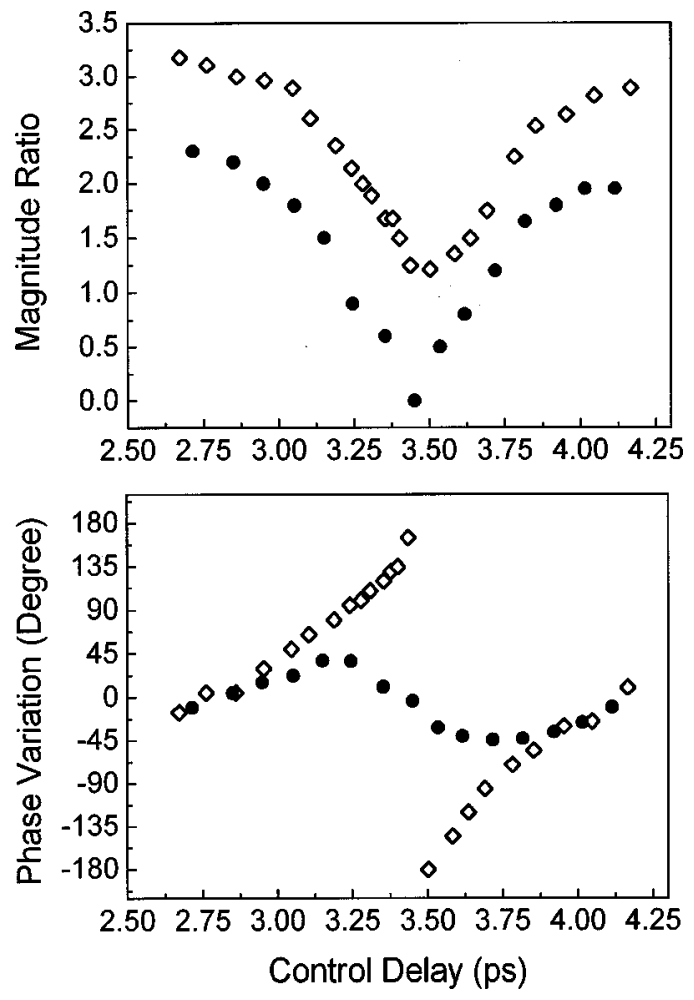

FIG. 4. Magntiude and phase variations of the initial oscillation after control excitation as a function of control delay for the $50 \AA$ InGaN MQW sample. The displacive force ratio between control/pump pulses was 2.5 (open diamonds) and 1.15 (solid circles).

With variable control pulse energy and time-delay, full $\pm 180^{\circ}$ phase shift can be achieved. Figure 4 shows the magnitude and phase variations of the initial oscillation due to control pulses as a function of the control pulse delay. The experiments were performed in $50 \AA \mathrm{MQW}$ sample. The control pulse intensity was kept fixed while the time delay between the pump and control pulses was varied from 2.76 to 4.14 ps (corresponding to $2.0-3.0$ oscillation periods). Open diamonds show the experimental data performed with an average pump power of $4.6 \mathrm{~mW}$ ( optical fluence of $3.6 \times 10^{-5} \mathrm{~J} / \mathrm{cm}^{2}$ ) and a step function ratio (control:pump) of 2.5 at a wavelength of $395 \mathrm{~nm}$. Oscillation magnitude variations between 3.3 and 1.2 times of the initial oscillation can be observed while phase shifts between $0^{\circ}$ and $\pm 180^{\circ}$ were achieved with different time delays.

The experiments were also performed by fixing the time delay between the pump and control pulses while changing the control/pump optical power ratio. With a stronger control pulse intensity, the magnitude and phase variations will both be larger. We were thus able to fine tune the control pulse delay and intensity in order to achieve the desired oscillation phase and magnitude control. However, if we kept on decreasing the control pulse energy, we will eventually not able to achieved $\pm 180^{\circ}$ phase variation control. For control delay at $180^{\circ}$ of the first induced cosine oscillations and with a control pulse energy much higher than the pump pulse energy, the oscillation after the control pulse excitation will follow the control-pulse-induced oscillation phase with an $180^{\circ}$ phase shift from the initial oscillation. If we kept on decreasing the control pulse energy at this specific time de- lay, the magnitude of the $180^{\circ}$ phase-shifted oscillation would also decrease and then reached a point of zero magnitude. The solid circles in Fig. 4 represent the experimental data corresponding to the earlier-mentioned zero-magnitude situation. The corresponding pump/control pulse energy and step response ratio are the same as those of Fig. 2. With a control delay of $3.45 \mathrm{ps}$ ( 2.5 oscillation periods), the control pulse was able to stop the induced acoustic phonon oscillation. However, with this control/pump ratio, the maximum phase shift between 2 and 3 oscillation period delay was only on the order of \pm 45 . With even decreased control pulse intensity, we were not only not able to cancel the oscillation or change the oscillation direction at 3.45 ps time delay, the amount of achievable phase variation at different time delay will also be smaller than that shown in Fig. 4.

In summary, after initiation of strong coherent acoustic phonon oscillations in InGaN/GaN multiple quantum wells, we demonstrated coherent control of the initiated coherent acoustic phonon oscillation. Both magnitude and phase manipulations can be achieved by controlling the intensity and time delay of an optical control pulse. This is different from most previous coherent control experiments where phase coherence or special engineering on the control pulse shape were required.

The authors would like to acknowledge stimulating scientific discussions with Christopher J. Stanton and Fabrice Vallée. The work is sponsored by National Science Council of Taiwan, Republic of China under Grant No. NSC 89-211M-002-082.

${ }^{1}$ G. C. Cho, W. Kutt, and H. Kurz, Phys. Rev. Lett. 65, 764 (1990).

${ }^{2}$ W. A. Kutt, W. Albrecht, and H. Kurz, IEEE J. Quantum Electron. 28, 2434 (1992).

${ }^{3}$ Y.-M. Chang, L. Xu, and H. W. K. Tom, Phys. Rev. B 59, 12220 (1999).

${ }^{4}$ T. Pfeifer, W. Kutt, H. Kurz, and H. Scholz, Phys. Rev. Lett. 69, 3248 (1992).

${ }^{5}$ T. K. Cheng, J. Vidal, H. J. Zeiger, G. Dresselhaus, M. S. Dresselhaus, and A. P. Ippen, Appl. Phys. Lett. 59, 1923 (1991).

${ }^{6}$ A. V. Kuznetsov and C. J. Stanton, Phys. Rev. B 51, 7555 (1995).

${ }^{7}$ A. Yamamoto, T. Mishina, and Y. Masumoto, Phys. Rev. Lett. 73, 740 (1994).

${ }^{8}$ A. Bartels, T. Dekorsy, H. Kurz, and K. Köhler, Phys. Rev. Lett. 82, 1044 (1999).

${ }^{9}$ K. Mizoguchi, M. Hase, and S. Nakashima, Phys. Rev. B 60, 8262 (1999).

${ }^{10}$ T. D. Krauss and F. W. Wise, Phys. Rev. Lett. 79, 5102 (1997).

${ }^{11}$ E. R. Thoen, G. Steinmeyer, P. Langlois, E. P. Ippen, G. E. Tudury, C. H. Brito Cruz, L. C. Barbosa, and C. L. Sedar, Appl. Phys. Lett. 73, 2149 (1998).

${ }^{12}$ N. Del Fatti, C. Voisin, D. Christofilos, F. Valleee, and C. Flytzanis, J. Phys. Chem. A 104, 4321 (2000).

${ }^{13}$ C.-K. Sun, J.-C. Liang, C. J. Stanton, A. Abare, L. Coldren, and S. P. DenBaars, Appl. Phys. Lett. 75, 1249 (1999).

${ }^{14}$ C.-K. Sun, J.-C. Liang, and X.-Y. Yu, Phys. Rev. Lett. 84, 179 (2000).

${ }^{15}$ A. M. Weiner, D. E. Leaird, G. P. Wiederrecht, and K. A. Nelson, Science 247, 1317 (1990).

${ }^{16}$ W. S. Warren, H. Rabitz, and M. Dahleh, Science 259, 1581 (1993).

${ }^{17}$ N. H. Bonadeo, J. Erland, D. Gammon, D. Park, D. S. Katzer, and D. G. Steel, Science 282, 1473 (1998).

${ }^{18}$ M. U. Wehner, M. H. Ulm, D. S. Chemla, and M. Wegner, Phys. Rev. Lett. 80, 1992 (1998).

${ }^{19}$ R. Atanasov, A. Haché, J. L. P. Hughes, H. M. van Driel, and J. E. Sipe, Phys. Rev. Lett. 76, 1703 (1996).

${ }^{20}$ P. C. M. Planken, I. Brener, M. C. Nuss, M. S. C. Luo, and S. L. Chuang, Phys. Rev. B 48, 4903 (1993).

${ }^{21}$ C.-K. Sun, J.-C. Liang, J.-C. Wang, F.-J. Kao, S. Keller, M. P. Mack, U. Mishra, and S. P. DenBaars, Appl. Phys. Lett. 76, 439 (2000). 